\title{
On critical exponents of a $k$-Hessian equation in the whole space
}

\author{
Yun WANG AND YUtian LeI \\ Institute of Mathematics, School of Mathematical Sciences, \\ Nanjing Normal University, Nanjing, 210023, China
}

\begin{abstract}
In this paper, we study negative classical solutions and stable solutions of the following $k$-Hessian equation

$$
F_{k}\left(D^{2} V\right)=(-V)^{p} \quad \text { in } R^{n}
$$

with radial structure, where $n \geq 3,1<k<n / 2$ and $p>1$. This equation is related to the extremal functions of the Hessian Sobolev inequality on the whole space. Several critical exponents including the Serrin type, the Sobolev type, and the Joseph-Lundgren type, play key roles in studying existence and decay rates. We believe that these critical exponents still come into play to research $k$-Hessian equations without radial structure. Keywords: $k$-Hessian equation, stable solution, critical exponent, Liouville theorem, decay rate
\end{abstract}

MSC2010: 35B33, 35J60

\section{Introduction}

In 1990, Tso [28 studied the relation between the value of exponent $p$ and the existence results for the $k$-Hessian equation $F_{k}\left(D^{2} V\right)=(-V)^{p}$ in bounded domains. The critical exponent $p=\frac{(n+2) k}{n-2 k}$ plays a key role. Those results are associated with the extremal functions of the Hessian Sobolev inequality for all $k$-admissible functions which was introduced by Wang in 32. Such an inequality with the critical exponent still holds in the whole space $R^{n}$, and the extremal functions are radially symmetric (cf. 5, 27]).

Consider the Euler-Lagrange equation

$$
F_{k}\left(D^{2} V\right)=(-V)^{p}, \quad V<0 \text { in } R^{n},
$$

with a general exponent $p>1$, where $n \geq 3,1<k<n / 2$. Here $F_{k}\left[D^{2} V\right]=$ $S_{k}\left(\lambda\left(D^{2} V\right)\right), \lambda\left(D^{2} V\right)=\left(\lambda_{1}, \lambda_{2}, \cdots, \lambda_{n}\right)$ with $\lambda_{i}$ being eigenvalues of the Hessian matrix $\left(D^{2} V\right)$, and $S_{k}(\cdot)$ is the $k$-th symmetric function:

$$
S_{k}(\lambda)=\sum_{1 \leq i_{1}<\cdots<i_{k} \leq n} \lambda_{i_{1}} \lambda_{i_{2}} \cdots \lambda_{i_{k}} .
$$

According to the conclusions in [1, $V<0$ ensures that the main part of (1.1) is elliptic. Namely, we always consider the $k$-admissible solutions in the cone

$$
\Phi^{k}:=\left\{u \in C^{2}\left(R^{n}\right) ; F_{s}\left(D^{2} V\right) \geq 0, s=1,2, \cdots, k\right\} .
$$

Such an equation does not only come into play to study the extremal functions of the Hessian Sobolev inequality, but also is helpful to investigate the global 
existence and blow-up in finite time span for the fully nonlinear parabolic equations (such as the equations studied in [12, 24] and [30]). tion

A special case is $F_{1}\left[D^{2} V\right]=\Delta V$, and (1.1) becomes the Lane-Emden equa-

$$
-\Delta u=u^{p}, \quad u>0 \text { in } R^{n} .
$$

The existence results of the solutions of this equation have provided an important ingredient in the study of conformal geometry, such as the extremal functions of the Sobolev inequalities and the prescribing scalar curvature problem. It was studied rather extensively. According to Theorem 3.41 in [21, (1.2) has no positive solution even on exterior domains when $p$ is not larger than the Serrin exponent (i.e. $p \in\left(1, \frac{n}{n-2}\right)$ ). The Liouville theorem in [9] shows that (1.2) has no positive classical solution in the subcritical case (i.e. $p \in\left[1, \frac{n+2}{n-2}\right)$ ). In the critical case (i.e. $p=\frac{n+2}{n-2}$ ), the positive classical solutions of (1.2) must be of the form

$$
u(x)=c\left(\frac{t}{t^{2}+\left|x-x^{*}\right|^{2}}\right)^{\frac{n-2}{2}}
$$

with constants $c, t>0$, and $x^{*} \in R^{n}$ (cf. [2]). In supercritical case (i.e. $\left.p>\frac{n+2}{n-2}\right)$, existence and asymptotic behavior of positive solutions are much complicated and not completely understood. In fact, we can find cylindrical shaped solutions which does not decay along some direction. In addition, there are radial solutions with the slow decay rates solving (1.2) (cf. [9, [13, 31] and many others). Furthermore, those radial solutions are of the form

$$
u(x)=\mu^{\frac{2}{p-1}} U(\mu|x|), \quad x \in R^{n},
$$

where $\mu=u^{\frac{p-1}{2}}(0)$, and $U(r)$ is the unique solution of

$$
\left\{\begin{array}{l}
-\left(U^{\prime \prime}+\frac{n-1}{r} U^{\prime}\right)=U^{p}, \quad U(r)>0, r>0 \\
U^{\prime}(0)=0, \quad U(0)=1 .
\end{array}\right.
$$

For the study of 'stable' positive solutions of (1.2), the Joseph-Lundgren exponent

$$
p_{j l}(n):=1+\frac{4}{n-4-2 \sqrt{n-1}}
$$

plays an important role (cf. [10]). Such an exponent is also essential to describe how the radial solutions intersect with the singular radial solution and with themselves (cf. [13). In addition, this Joseph-Lundgren exponent can be used to study the Morse index for the sign-changed solutions of the Lane-Emden equation (cf. 8]) and other nonlinear elliptic equations with supercritical exponents (cf. 6] 7] and [1]).

In this paper, our purpose is to study the relation between the critical exponents and existence of kinds of solutions of $k$-Hessian equation (1.1). As the beginning of the study, we are concerned about the increasing negative solution of (1.1) with radial structure as in [5 and 20. Thus, (1.1) is reduced to the following equation

$$
-\frac{1}{k} C_{n-1}^{k-1}\left(r^{n-k}\left|u^{\prime}\right|^{k-1} u^{\prime}\right)^{\prime}=r^{n-1} u^{p}, \quad u(r)>0 \quad \text { as } \quad r>0 .
$$

Here $u(r)=u(|x|)=-V(x), n \geq 3,1<k<n / 2$ and $p>1$. In fact, in the critical case (i.e. $p=\frac{(n+2) k}{n-2 k}$ ), the extremal functions of the Hessian Sobolev 
inequality are radially symmetric (cf. [5, 27] and [32]). In the noncritical case, it is clearer and more concise to study the critical exponents of the radial solutions. We believe that the ideas are helpful to investigate the corresponding problems of the solutions with general form, and those critical exponents still come into play in the study of $k$-Hessian equations without radial structure.

\section{$1.1 \quad$ Regular solutions}

Clearly, (1.4) has a singular solution

$$
u_{s}(r)=A r^{-\frac{2 k}{p-k}}, \quad \text { with } \quad A:=\left(\frac{1}{k} C_{n-1}^{k-1}\right)^{\frac{1}{p-k}}\left(\frac{2 k}{p-k}\right)^{\frac{k}{p-k}}\left(n-\frac{2 p k}{p-k}\right)^{\frac{1}{p-k}}
$$

If write $V(x)=-u_{s}(|x|)$, then $V(x)$ only belong to $C^{2}\left(R^{n} \backslash\{0\}\right)$ (even it does not belong to $\left.L_{l o c}^{\infty}\left(R^{n}\right)\right)$.

We are mainly concerned with the $k$-admissible solutions of (1.1). Consider the following boundary values problem

$$
\left\{\begin{array}{l}
-\frac{1}{k} C_{n-1}^{k-1}\left(r^{n-k}\left|u^{\prime}\right|^{k-1} u^{\prime}\right)^{\prime}=r^{n-1} u^{p}, \quad u(r)>0, r>0 \\
u^{\prime}(0)=0, \quad u(0)=\rho\left(:=\mu^{\frac{2 k}{p-k}}\right)>0 .
\end{array}\right.
$$

Definition 1.1. If a solution $u(r)$ of (1.6) satisfies $u(|x|) \in C^{2}\left(R^{n}\right)$, then $u(r)$ is called a regular solution.

Recall two critical exponents: Serrin exponent $p_{s e}:=\frac{n k}{n-2 k}$, and Sobolev exponent $p_{s o}:=\frac{(n+2) k}{n-2 k}$.

When $p$ is not larger than the Serrin exponent, (1.1) has no negative $k$ admissible solution (cf. [15], 22] and [23]). Thus, we always assume in this paper that $p$ is larger than the Serrin exponent

$$
p>p_{\text {se }} .
$$

In the critical case (i.e. $p=p_{s o}$ ), all the regular solutions of (1.6) can be written as the explicit form (cf. Remark 1.4 in [20])

$$
u_{\rho}(r)=\left(\frac{1}{k} C_{n-1}^{k-1}\right)^{\frac{1}{p-k}} \rho\left(1+\frac{k}{n^{1 / k}(n-2 k)}\left(\rho^{\frac{k+1}{n-2 k}} r\right)^{2}\right)^{-\frac{n-2 k}{2 k}} .
$$

Therefore, we will be concerned with the noncritical cases.

Theorem 1.1. When $p<p_{s o}$, (1.6) has no regular solution.

Remark 1.1. By a direct calculation, when $p_{s e}<p<p_{s o}$, besides $u_{s}$ given by (1.5), (1.4) has other singular solutions $U_{s}(r)$ satisfying $U_{s}(r) / u_{s}(r) \rightarrow 1$ as $r \rightarrow 0$ and $U_{s}(r) r^{\frac{n-2 k}{k}} \rightarrow \lambda>0$ as $r \rightarrow \infty$. When $k=1$, this result can be found in [9], [13], 31.

Theorem 1.2. When $p>p_{\text {so }}$, all the positive regular solution $u_{\mu}$ of (1.6) satisfies $u_{\mu}(r) \simeq r^{-\frac{2 k}{p-k}}$ for large $r$. Furthermore, they are the form of

$$
u_{\mu}(r)=\mu^{\frac{2 k}{p-k}} u_{1}(\mu r), \quad r \geq 0,
$$


where $u_{1}(r)$ is the solution of

$$
\left\{\begin{array}{l}
-\frac{1}{k} C_{n-1}^{k-1}\left(r^{n-k}\left|u^{\prime}\right|^{k-1} u^{\prime}\right)^{\prime}=r^{n-1} u^{p}, \quad u(r)>0, r>0 \\
u^{\prime}(0)=0, \quad u(0)=1 .
\end{array}\right.
$$

Here, $u(r) \simeq r^{-\theta}$ means that there exists $C>1$ such that $\frac{1}{C} \leq u(r) r^{\theta} \leq C$ for large $r$.

Remark 1.2. Problem (1.10) has a entire solution when $p>p_{\text {so }}$. In fact, by a standard argument of contraction, (1.10) has a unique local positive solution $u$ (cf. Proposition 2.1 in 20]). There holds $u^{\prime}<0$ as long as $u>0$ (see the proof of Lemma 2.1). Extend this local solution rightwards. Then $u>0$ for all $r>0$. Otherwise, it contradicts with the Liouville theorem in [28].

\subsection{Stable solutions}

Definition 1.2. We say that a positive solution $u \in C^{1}(0, \infty)$ of (1.4) is stable if

$$
\begin{gathered}
\int_{0}^{\infty}\left[\frac{1}{k} C_{n-1}^{k-1} r^{n-k}\left|u^{\prime}\right|^{k-1} u^{\prime} \varphi^{\prime}-r^{n-1} u^{p} \varphi\right] d r=0 ; \\
Q_{u}(\varphi):=C_{n-1}^{k-1} \int_{0}^{\infty} r^{n-k}\left|u^{\prime}\right|^{k-1}\left(\varphi^{\prime}\right)^{2} d r-p \int_{0}^{\infty} r^{n-1} u^{p-1} \varphi^{2} d r \geq 0
\end{gathered}
$$

for all $\varphi \in W_{*}$, where $W_{*}=\left\{\varphi(r) ; \varphi(r)=\phi(x) \in C_{c}^{\infty}\left(R^{n}\right), r=|x|\right\}$.

Similarly, a positive solution $u \in C^{1}(0, \infty)$ of $(1.4)$ is stable on a set $(R, \infty)$ for some $R>0$, if (1.11) holds for all $\varphi \in W_{*}$, and (1.12) holds for all $\varphi \in$ $C_{c}^{\infty}(R, \infty)$.

Indeed, the fact that the first order Fréchet derivative of the functional $J(u)$ is equal to zero and the second order Fréchet derivative is nonnegative can lead to this definition, where

$$
J(u)=\frac{C_{n-1}^{k-1}}{k(k+1)} \int_{0}^{\infty}\left|u^{\prime}\right|^{k+1} r^{n-k} d r-\frac{1}{p+1} \int_{0}^{\infty} u^{p+1} r^{n-1} d r .
$$

In addition, $Q_{u}(\varphi) \geq 0$ can also be obtained by linearizing (1.4).

It is not difficult to verify that the regular solutions $u_{\rho}$ given by (1.8) and $u_{\mu}$ given by (1.9) satisfy (1.11). For the singular solution $u_{s}$ expressed by (1.5), $p>p_{s e}$ implies that 0 is not the singular point in integral terms of (1.11) (see the proof of Theorem 1.4). Therefore, $u_{s}$ also satisfies (1.11).

Recall other two critical exponents: the Joseph-Lundgren exponent

$$
p_{j l}= \begin{cases}\infty, & \text { if } N \leq 2 k+8, \\ \frac{k\left[n^{2}-2(k+3) n+4 k\right]+4 k \sqrt{2(k+1) n-4 k}}{(n-2 k)(n-2 k-8)}, & \text { if } N>2 k+8 ;\end{cases}
$$

and

$$
p^{*}=k \frac{n+2 k}{n-2 k} \text {. }
$$


Clearly, $p_{s e}<p_{s o}<p_{j l}$. In addition, $p_{s o}<p^{*}$ by virtue of $1<k<n / 2$. In view of $2 k\left(k^{2}+6 k+1\right) /(k-1)^{2}>2 k+8$, we can deduce the relation between $p^{*}$ and $p_{j l}$ as follows

$$
\begin{aligned}
& p^{*} \geq p_{j l}, \quad \text { if } n \geq 2 k\left(k^{2}+6 k+1\right) /(k-1)^{2} \\
& p^{*}<p_{j l}, \quad \text { if } n<2 k\left(k^{2}+6 k+1\right) /(k-1)^{2}
\end{aligned}
$$

Under the scaling transformation, $p=p_{\text {so }}$ ensures that equation (1.1) and energy $\|\cdot\|_{p+1}$ are invariant (cf [15]), and $p=p^{*}$ ensures that equation (1.1) and energy $\|\cdot\|_{p+k}$ are invariant (cf [17]). In addition, $p^{*}$ is essential to study the separation property of solutions (see the following Remark).

Remark 1.3. Let $u_{\mu}(r)$ be a regular solution of (1.6). Corollary 1.7 in [20] implies that, when $p \geq \max \left\{p^{*}, p_{j l}\right\}, u_{\mu}(r)<u_{s}(r)$ for $r>0$, and $u_{\mu_{1}}(r)<$ $u_{\mu_{2}}(r)$ for $r>0$ as long as $\mu_{1}<\mu_{2}$.

The exponent $p^{*}$ also appears in the study of $\gamma$-Laplace equations (cf. [16] and [20]) and integral equations involving Wolff potentials (cf. [3], [19], 26] and [29]). In particular, it plays an important role to investigate integrability, decay rates and intersection properties of the positive entire solutions. In addition, this exponent ensures that equation and energy $\|\cdot\|_{p+\gamma-1}$ are invariant under the scaling transformation (cf [17]).

In particular, for the $\gamma$-Laplace equation

$$
-\operatorname{div}\left(|\nabla u|^{\gamma-2} \nabla u\right)=K(x) u^{p}, \quad u>0 \quad \text { in } R^{n}
$$

we write $p_{s e}(\gamma)=\frac{n(\gamma-1)}{n-\gamma}, p_{s o}(\gamma)=\frac{n \gamma}{n-\gamma}-1, p^{*}(\gamma)=\frac{n+\gamma}{n-\gamma}(\gamma-1), p_{j l}=\gamma-1+$ $\gamma^{2}[n-\gamma-2-2 \sqrt{(n-1) /(\gamma-1)}]^{-1}$ as $n>\frac{\gamma(\gamma+3)}{\gamma-1}$, and $p_{j l}=\infty$ as $n \leq \frac{\gamma(\gamma+3)}{\gamma-1}$.

If $\gamma \in(1,2), p_{s e}(\gamma)<p^{*}(\gamma)<p_{s o}(\gamma)$. When $K(x) \equiv 1$, according to the Liouville theorem in [25], (1.13) has no positive solution as $p<p_{s o}(\gamma)$, and $p^{*}(\gamma)$ does not make sense. When $K(x)$ is a double bounded function, according to the result in [17, (1.13) has positive radial solutions as long as $p>p_{s e}(\gamma)$. Now, $p^{*}$ comes into play in studying integrability and decay rates of positive solutions.

Now, we state the results about the stable solutions.

Theorem 1.3. When $p<p_{j l}$, 1.4) has no stable solution.

Theorem 1.4. When $p \geq p_{j l}$, the singular solution $u_{s}$ given by (1.5) is a stable solution of (1.4).

Theorem 1.5. When $p=p_{\text {so }}$ or $p \geq \max \left\{p^{*}, p_{j l}\right\}$, all the regular solutions of (1.6) are stable solutions of (1.4) on $(R, \infty)$ for some $R>0$. When $p_{\text {se }}<p<$ $p_{\text {so }}$, the singular solutions introduced in Remark 1.1 are stable solution of (1.4) on $(R, \infty)$ for some $R>0$.

Remark 1.4. Theorem 1.4 shows that $u_{s}$ is also a stable solution of (1.4) on $(R, \infty)$ for some $R>0$ when $p \geq p_{j l}$. Combining with Theorem 1.5, we know that (1.4) has stable solutions on $(R, \infty)$ for some $R>0$ when $p \in$ $\left(p_{s e}, p_{s o}\right] \cup\left[p_{j l}, \infty\right)$. To our knowledge, it is unknown whether (1.4) has no stable solution on $(R, \infty)$ for some $R>0$ when $p$ belongs to the gap $\left(p_{s o}, p_{j l}\right)$. 


\section{Regular solutions}

Lemma 2.1. Let $u$ be a regular solution of (1.6). Then, $u^{\prime}<0$ for $r>0$, and $u(r) \rightarrow 0$ as $r \rightarrow \infty$. Moreover, there are positive constants $C_{1}, C_{2}$ such that for large $r$,

$$
C_{1} r^{-\frac{n-2 k}{k}} \leq u(r) \leq C_{2} r^{-\frac{2 k}{p-k}} .
$$

Proof. Step 1. Since $u$ is a positive solution of (1.4),

$$
-\frac{1}{k} C_{n-1}^{k-1}\left(r^{n-k}\left|u^{\prime}\right|^{k-1} u^{\prime}\right)^{\prime}>0, \quad r>0 .
$$

Integrating from 0 to $R$ with $R>0$, we obtain

$$
R^{n-k}\left|u^{\prime}(R)\right|^{k-1} u^{\prime}(R)<0
$$

and hence $u^{\prime}<0$ is verified.

Step 2. By $u>0$ and $u^{\prime}<0$ for $r>0$, we know that $\lim _{r \rightarrow \infty} u(r)$ exists and hence is nonnegative. Suppose that $\lim _{r \rightarrow \infty} u(r)>0$, then there exists a constant $c>0$ such that $u \geq c$, and hence

$$
-\frac{1}{k} C_{n-1}^{k-1}\left(r^{n-k}\left|u^{\prime}\right|^{k-1} u^{\prime}\right)^{\prime} \geq c^{p} r^{n-1} .
$$

Integrating from 0 to $R$, we obtain

$$
R^{n-k}\left|u^{\prime}(R)\right|^{k-1} u^{\prime}(R) \leq-C R^{n} .
$$

Here $C>0$ is independent of $R$. This result, together with $u^{\prime}<0$, implies $u^{\prime}(R) \leq-C R$. Integrating again yields

$$
u(r) \leq u(0)-C r^{2} .
$$

Letting $r \rightarrow \infty$, we see a contradiction with $u>0$. This shows that $u(r) \rightarrow 0$ as $r \rightarrow \infty$.

Step 3. According to the results in [14] or [23], the regular solution of (1.6) satisfies

$$
c_{1} W_{\frac{2 k}{k+1}, k+1}\left(u^{p}\right)(x) \leq u(|x|) \leq c_{2}\left[\inf _{x \in R^{n}} u(|x|)+W_{\frac{2 k}{k+1}, k+1}\left(u^{p}\right)(x)\right],
$$

where $c_{1}, c_{2}$ are positive constant, and $W_{\frac{2 k}{k+1}, k+1}\left(u^{p}\right)$ is the Wolff potential of $u^{p}$. Namely,

$$
W_{\frac{2 k}{k+1}, k+1}\left(u^{p}\right)(x)=\int_{0}^{\infty}\left(\frac{\int_{B_{t}(x)} u^{p}(|y|) d y}{t^{n-2 k}}\right)^{\frac{1}{k}} \frac{d t}{t} .
$$

Therefore, for large $|x|$,

$$
u(|x|) \geq c \int_{|x|+1}^{\infty}\left(\frac{\int_{B_{1}(0)} u^{p}(|y|) d y}{t^{n-2 k}}\right)^{\frac{1}{k}} \frac{d t}{t} \geq c \int_{|x|+1}^{\infty} t^{\frac{2 k-n}{k}} \frac{d t}{t}=c|x|^{\frac{2 k-n}{k}} .
$$

Since $u$ is radially symmetric and decreasing, we can also get

$$
u(|x|) \geq c \int_{0}^{|x| / 2}\left(\frac{\int_{B_{|x|}(0) \cap B_{t}(x)} u^{p}(|y|) d y}{t^{n-2 k}}\right)^{\frac{1}{k}} \frac{d t}{t} \geq c u^{\frac{p}{k}}(|x|)|x|^{2},
$$

which implies that $u(|x|) \leq c|x|^{\frac{2 k}{k-p}}$ for large $|x|$.

The proof is complete. 


\subsection{Proof of Theorem 1.1}

Let $p<p_{s o}$. Assume that (1.6) has a positive regular solution $u$, we will deduce a contradiction.

Step 1. By (2.1), there exists $R>0$ such that $u(r) \leq C r^{-\frac{2 k}{p-k}}$ for $r>R$. Thus,

$$
\int_{0}^{\infty} r^{n-1} u^{p+1} d r \leq C(R)+\int_{R}^{\infty} r^{n-\frac{2(p+1) k}{p-k}} \frac{d r}{r}<\infty .
$$

Step 2. Let $\varphi \in C^{\infty}(0, \infty)$ satisfy $\varphi(r)=1$ when $r \in(0,1], \varphi(r)=0$ when $r \in[2, \infty)$, and $0 \leq \varphi \leq 1$. Write $\varphi_{R}(r)=\varphi\left(\frac{r}{R}\right)$. Multiply (1.4) by $u \varphi_{R}^{k+1}$ and integrate on $(0, \infty)$. By the initial value condition in (1.6), we get

$$
\begin{aligned}
\int_{0}^{\infty} r^{n-k}\left|u^{\prime}\right|^{k+1} \varphi_{R}^{k+1} d r & =\frac{k}{C_{n-1}^{k-1}} \int_{0}^{\infty} r^{n-1} u^{p+1} \varphi_{R}^{k+1} d r \\
& -(k+1) \int_{0}^{\infty} r^{n-k} u \varphi_{R}^{k}\left|u^{\prime}\right|^{k-1} u^{\prime} \varphi_{R}^{\prime} d r
\end{aligned}
$$

By the Young inequality and the Hölder inequality, for a small $\epsilon>0$, there holds that

$$
\begin{gathered}
\left.\left.\left|\int_{0}^{\infty} r^{n-k} u \varphi_{R}^{k}\right| u^{\prime}\right|^{k-1} u^{\prime} \varphi_{R}^{\prime} d r\left|\leq \epsilon \int_{0}^{\infty} r^{n-k}\right| u^{\prime}\right|^{k+1} \varphi_{R}^{k+1} d r \\
+\frac{C_{\epsilon}}{R^{k+1}}\left(\int_{0}^{\infty} r^{n-1} u^{p+1} d r\right)^{\frac{k+1}{p+1}}\left(\int_{R}^{2 R} r^{\theta+1} \frac{d r}{r}\right)^{\frac{p-k}{p+1}}
\end{gathered}
$$

where $\frac{p-k}{p+1} \theta=n-k-(n-1) \frac{k+1}{p+1}$. Therefore, $(\theta+1) \frac{p-k}{p+1}-(k+1)=n \frac{p-k}{p+1}-2 k<0$ by virtue of $p<p_{\text {so. }}$. Letting $R \rightarrow \infty$, we deduce from (2.3), (2.4) and (2.5) that

$$
\int_{0}^{\infty} r^{n-k}\left|u^{\prime}\right|^{k+1} d r<\infty
$$

Step 3. Multiplying (1.4) by $u$ and integrating on $(0, R)$, we obtain that

$$
\int_{0}^{R} r^{n-k}\left|u^{\prime}\right|^{k+1} d r-R^{n-k} u(R)\left|u^{\prime}(R)\right|^{k-1} u^{\prime}(R)=\frac{k}{C_{n-1}^{k-1}} \int_{0}^{R} r^{n-1} u^{p+1} d r .
$$

By (2.6) and (2.3), there exists $R_{j} \rightarrow \infty$ such that

$$
R_{j}^{n-k+1}\left|u^{\prime}\left(R_{j}\right)\right|^{k+1}+R_{j}^{n} u^{p+1}\left(R_{j}\right) \rightarrow 0 .
$$

Therefore, by $p<p_{\text {so }}$,

$$
R_{j}^{n-k} u\left(R_{j}\right)\left|u^{\prime}\left(R_{j}\right)\right|^{k-1} u^{\prime}\left(R_{j}\right) \rightarrow 0, \quad \text { as } R_{j} \rightarrow \infty .
$$

Inserting this result into (2.7) and letting $R=R_{j} \rightarrow \infty$, we obtain

$$
\int_{0}^{\infty} r^{n-k}\left|u^{\prime}\right|^{k+1} d r=\frac{k}{C_{n-1}^{k-1}} \int_{0}^{\infty} r^{n-1} u^{p+1} d r
$$

Step 4. Multiplying (1.4) by $r u^{\prime}$ and integrating on $(0, R)$, we have the Pohozaev type equality

$$
\begin{aligned}
& -\frac{n-2 k}{k+1} \int_{0}^{R} r^{n-k}\left|u^{\prime}\right|^{k+1} d r+\frac{k}{C_{n-1}^{k-1}} \frac{n}{p+1} \int_{0}^{R} r^{n-1} u^{p+1} d r \\
& =\frac{k}{k+1} R^{n-k+1}\left|u^{\prime}(R)\right|^{k+1}+\frac{k}{(p+1) C_{n-1}^{k-1}} R^{n} u^{p+1}(R) .
\end{aligned}
$$


By (2.8), the right hand side of (2.10) converges to zero when $R=R_{j} \rightarrow \infty$. Letting $R=R_{j} \rightarrow \infty$ in (2.10) and using (2.9), we can see $\frac{n-2 k}{k+1}=\frac{n}{p+1}$, which contradicts with $p<p_{\text {so }}$.

\subsection{Proof of Theorem 1.2}

When $k=1$, the proof of the slow decay is based on the comparison principle (cf. Lemma 2.20 and Theorem 2.25 in [18). For the quasilinear equation (1.4), we use the monotony inequality replacing the comparison principle.

Lemma 2.2. Let $u(r)$ be a regular solution of (1.6). If $u(r)=O\left(r^{-\frac{2 k}{p-k}-\varepsilon}\right)$ with some $\varepsilon \in\left(0, \frac{n-2 k}{k}-\frac{2 k}{p-k}\right)$ for large $r$, then $u(r)=O\left(r^{(2 k-n) / k}\right)$ for large $r$.

Proof. If $u(r)=O\left(r^{-\frac{2 k}{p-k}-\varepsilon}\right)$ for large $r$, we can find a large $R>0$ such that as $r>R$

$$
u(r) \leq C r^{-\frac{2 k}{p-k}-\varepsilon} .
$$

By Lemma 2.1, $\inf _{[0, \infty)} u(r)=0$. Using (2.2) we have

$$
u(|x|) \leq C\left(I_{1}+I_{2}+I_{3}\right),
$$

where

$$
\begin{gathered}
I_{1}=\int_{0}^{\frac{|x|}{2}}\left(\frac{\int_{B_{t}(x)} u^{p}(|y|) d y}{t^{n-2 k}}\right)^{\frac{1}{k}} \frac{d t}{t}, \\
I_{2}=\int_{\frac{|x|}{2}}^{\infty}\left(\frac{\int_{B_{t}(x) \cap B_{R}(0)} u^{p}(|y|) d y}{t^{n-2 k}}\right)^{\frac{1}{k}} \frac{d t}{t}, \\
I_{3}=\int_{\frac{|x|}{2}}^{\infty}\left(\frac{\int_{B_{t}(x) \backslash B_{R}(0)} u^{p}(|y|) d y}{t^{n-2 k}}\right)^{\frac{1}{k}} \frac{d t}{t} .
\end{gathered}
$$

For sufficiently large $|x|$, we can deduce from (2.11) that

$$
\begin{aligned}
& I_{1} \leq C|x|^{-\frac{p}{k}\left(\frac{2 k}{p-k}+\varepsilon\right)} \int_{0}^{\frac{|x|}{2}}\left(\frac{\int_{B_{t}(x)} d y}{t^{n-2 k}}\right)^{\frac{1}{k}} \frac{d t}{t} \leq C|x|^{-\frac{2 k}{p-k}-\varepsilon \frac{p}{k}}, \\
& I_{2} \leq C\left(\left|B_{R}(0)\right| u^{p}(0)\right)^{1 / k} \int_{\frac{|x|}{2}}^{\infty} t^{\frac{2 k-n}{k}} \frac{d t}{t} \leq C|x|^{-\frac{n-2 k}{k}}, \\
& I_{3} \leq \int_{\frac{|x|}{2}}^{\infty}\left(\frac{\int_{B_{t+|x|}(0) \backslash B_{R}(0)} u^{p}(|y|) d y}{t^{n-2 k}}\right)^{\frac{1}{k}} \frac{d t}{t} \leq C|x|^{-\frac{2 k}{p-k}-\varepsilon \frac{p}{k}} .
\end{aligned}
$$

These estimates show that $u(r) \leq C\left(r^{-\frac{n-2 k}{k}}+r^{-\frac{2 k}{p-k}-\varepsilon \frac{p}{k}}\right) \leq C r^{-\frac{2 k}{p-k}-\varepsilon \frac{p}{k}}$. Replacing (2.11) by this result to estimate $I_{1}, I_{2}$ and $I_{3}$ as we have done above, we get

$$
u(r) \leq C\left(r^{-\frac{n-2 k}{k}}+r^{-\frac{2 k}{p-k}-\varepsilon\left(\frac{p}{k}\right)^{2}}\right) \leq C r^{-\frac{2 k}{p-k}-\varepsilon\left(\frac{p}{k}\right)^{2}} .
$$

By iterating $m$ times, we can obtain

$$
u(r) \leq C\left(r^{-\frac{n-2 k}{k}}+r^{-\frac{2 k}{p-k}-\varepsilon\left(\frac{p}{k}\right)^{m}}\right) .
$$

Clearly, there exists a sufficiently large $m_{0}$ such that $\frac{n-2 k}{k} \leq \frac{2 k}{p-k}+\varepsilon\left(\frac{p}{k}\right)^{m_{0}}$. Thus, after $m_{0}$ steps, we derive that,

$$
u(r) \leq C r^{-\frac{n-2 k}{k}} \text { for large } r .
$$

Lemma 2.2 is proved. 
Lemma 2.3. Let $u(r)$ be a regular solution of (1.6). If $u(r)=o\left(r^{-\frac{2 k}{p-k}}\right)$ for large $r$, then $u(r)=O\left(r^{(2 k-n) / k}\right)$ for large $r$.

Proof. Step 1. Let $\varphi(r) \in C^{1}(0, \infty)$ satisfy $\lim _{r \rightarrow \infty} r^{n-\frac{2 p k}{p-k}} \varphi(r)=0$. Integrating (1.4) from 0 to $r$, we have

$$
\left|u^{\prime}\right|^{k-1} u^{\prime}=-\frac{k}{C_{n-1}^{k-1}} r^{k-n} \int_{0}^{r} s^{n-1} u^{p}(s) d s .
$$

Thus, by $u(r)=o\left(r^{-\frac{2 k}{p-k}}\right)$ when $r \rightarrow \infty$, it follows that

$$
r^{n-k}\left|u^{\prime}(r)\right|^{k} \varphi(r) \rightarrow 0 .
$$

Multiply (1.4) by $\varphi$ and integrate from $R$ to $\infty$. By (2.13), we obtain that

$$
\begin{aligned}
& \int_{R}^{\infty} r^{n-k}\left|u^{\prime}\right|^{k-1}\left(u^{\prime}\right) \varphi^{\prime} d r \\
& =-R^{n-k}\left|u^{\prime}(R)\right|^{k-1} u^{\prime}(R) \varphi(R)+\frac{k}{C_{n-1}^{k-1}} \int_{R}^{\infty} r^{n-1} u^{p} \varphi d r
\end{aligned}
$$

Write $h(r):=c_{*} r^{-\theta}$, where $c_{*}$ is a positive constant determined later, and $\theta:=\frac{2 k}{p-k}+\epsilon_{0}$ with suitably small $\epsilon_{0}>0$. By simply calculating and integrating by parts, we get

$$
\begin{aligned}
& \int_{R}^{\infty} r^{n-k}\left|h^{\prime}\right|^{k-1} h^{\prime} \varphi^{\prime} d r=-\left(c_{*} \theta\right)^{k} \int_{R}^{\infty} r^{n-k(\theta+2)} \varphi^{\prime} d r \\
& =\left(c_{*} \theta\right)^{k}[n-k(\theta+2)] \int_{R}^{\infty} r^{n-1-k(\theta+2)} \varphi d r+\left(c_{*} \theta\right)^{k} R^{n-k(\theta+2)} \varphi(R) .
\end{aligned}
$$

Subtracting this result from (2.14) yields

$$
\begin{aligned}
& \int_{R}^{\infty} r^{n-k}\left[\left|u^{\prime}\right|^{k-1} u^{\prime}-\left|h^{\prime}\right|^{k-1} h^{\prime}\right] \varphi^{\prime} d r \\
& =\left[\left(c_{*} \theta\right)^{k} R^{n-k(\theta+2)}+R^{n-k}\left|u^{\prime}(R)\right|^{k-1} u^{\prime}(R)\right] \varphi(R) \\
& +\int_{R}^{\infty} r^{n-1}\left[\frac{k u^{p}}{C_{n-1}^{k-1}}-\frac{\left(c_{*} \theta\right)^{k}[n-k(\theta+2)]}{r^{k(\theta+2)}}\right] \varphi d r .
\end{aligned}
$$
that

Step 2. In view of $k(\theta+2)=\frac{2 p k}{p-k}+k \epsilon_{0}$, we can find $\eta_{0} \in\left(0, k \epsilon_{0} / p\right)$ such

$$
k(\theta+2)>\frac{2 p k}{p-k}+p \eta_{0} .
$$

Since $u \in C^{2}$ is decreasing and $u(r)=o\left(r^{-\frac{2 k}{p-k}}\right)$ for large $r$, then either there exist positive constants $c_{1}, c_{2}$ such that

$$
c_{1} r^{-\frac{2 k}{p-k}} \geq u(r) \geq c_{2} r^{-\frac{2 k}{p-k}-\eta_{0}}
$$

when $r$ is suitably large, or $\lim _{r \rightarrow \infty} u(r) r^{\frac{2 k}{p-k}+\eta_{0}}=0$, which implies that there exists $\eta \in\left(0, \eta_{0}\right)$ such that for large $r$,

$$
u(r) \leq c r^{-\frac{2 k}{p-k}-\left(\eta_{0}-\eta\right)}
$$


If (2.18) is true, Lemma 2.3 can be proved easily by Lemma 2.2

In the following, we assume that (2.17) is true. Take $\varphi=r^{-m}(u-h)_{+}$in (2.15), where $m>n-\frac{2 p k}{p-k}$ is sufficiently large. Then,

$$
\begin{aligned}
& \int_{R}^{\infty} r^{n-k-m}\left[\left|u^{\prime}\right|^{k-1} u^{\prime}-\left|h^{\prime}\right|^{k-1} h^{\prime}\right]\left[(u-h)_{+}\right]^{\prime} d r \\
& =\left[\left(c_{*} \theta\right)^{k} R^{n-k(\theta+2)-m}+R^{n-k-m}\left|u^{\prime}(R)\right|^{k-1} u^{\prime}(R)\right][u(R)-h(R)]_{+} \\
& +\int_{R}^{\infty} r^{n-m-1}\left[\frac{k u^{p}}{C_{n-1}^{k-1}}-\frac{\left(c_{*} \theta\right)^{k}[n-k(\theta+2)]}{r^{k(\theta+2)}}\right](u-h)_{+} d r \\
& +m \int_{R}^{\infty} r^{n-k-m-1}\left[\left|u^{\prime}\right|^{k-1} u^{\prime}-\left|h^{\prime}\right|^{k-1} h^{\prime}\right](u-h)_{+} d r .
\end{aligned}
$$

By (2.12), (2.17) and (2.16), for any $\delta \in(0,1)$, we can find $R_{0}>0$ such that as $r \geq R_{0},\left|h^{\prime}\right|^{k} \leq \delta\left|u^{\prime}\right|^{k}$. Therefore, the last term of the right hand side of (2.19) with $R=R_{0}$ is not larger than $m(1-\delta) \int_{R_{0}}^{\infty} r^{n-k-m-1}\left|u^{\prime}\right|^{k-1} u^{\prime}(u-h)_{+} d r$. Choose $c_{*}=u\left(R_{0}\right) R_{0}^{\theta}$ to ensure $u\left(R_{0}\right)=h\left(R_{0}\right)$. Therefore, the first term of the right hand side of (2.19) with $R=R_{0}$ is zero. Thus, from (2.19) with $R=R_{0}$ it follows that

$$
\begin{aligned}
& \int_{R_{0}}^{\infty} r^{n-k-m}\left[\left|u^{\prime}\right|^{k-1} u^{\prime}-\left|h^{\prime}\right|^{k-1} h^{\prime}\right]\left[(u-h)_{+}\right]^{\prime} d r \\
& \leq \int_{R_{0}}^{\infty} r^{n-m-1}\left[\frac{k u^{p}}{C_{n-1}^{k-1}}-m(1-\delta) r^{-k}\left|u^{\prime}\right|^{k}\right](u-h)_{+} d r .
\end{aligned}
$$

By (2.12) and the monotonicity of $u(r)$, there holds

$$
r^{-k}\left|u^{\prime}(r)\right|^{k} \geq \frac{k}{C_{n-1}^{k-1}} r^{-n} u^{p}(r) \int_{0}^{r} s^{n-1} d s \geq \frac{k u^{p}(r)}{n C_{n-1}^{k-1}} .
$$

Taking $m$ suitably large, we obtain that the right hand side of (2.20) is not larger than zero. In view of the monotony inequality $\left(|a|^{k-1} a-|b|^{k-1} b\right)(a-b) \geq$ $2^{k-1}|a-b|^{k+1}$, we obtain from (2.20) that

$$
\int_{R_{0}}^{\infty} r^{n-k}\left(\left[(u-h)_{+}\right]^{\prime}\right)^{k+1} d r \leq 0,
$$

which implies $[u(r)-h(r)]_{+} \equiv$ Constant for $r \geq R_{0}$. In view of $u\left(R_{0}\right)=h\left(R_{0}\right)$, it follows Constant $=0$, which implies $u(r) \leq h(r)$ for $r \geq R_{0}$. Applying Lemma 2.2. we can also see the conclusion of Lemma 2.3.

Proof of Theorem 1.2. Let $p>p_{s o}$.

Step 1. By Lemma 2.1, we see that $u(r) \leq C r^{-\frac{2 k}{p-k}}$ for large $r$. We claim that there exists $c>0$ such that $u(r) \geq c r^{-\frac{2 k}{p-k}}$ for large $r$.

Otherwise, $\lim _{r \rightarrow \infty} u(r) r^{\frac{2 k}{p-k}}=0$. By Lemma 2.3 it follows that $u(r)=$ $O\left(r^{\frac{2 k-n}{k}}\right)$ for large $r$. Thus, $V(x) \in L^{p+1}\left(R^{n}\right) \cap C^{2}\left(R^{n}\right)$ (here $V(x)=-u(|x|)$ ). According to Theorem 4.4 in [15], we know $p=p_{\text {so }}$, which contradicts with $p>p_{\text {so }}$.

Step 2. We define by scaling a new function

$$
w(r)=\mu^{\frac{2 k}{p-k}} u(\mu r), \quad \mu>0 .
$$


By a direct calculation, we see that $w$ still satisfies (1.4). Applying the initial value conditions, we can obtain the second conclusion of Theorem 1.2 .

Remark 2.1. Let $u_{\mu}(r)$ be a regular solution of (1.6) with $p>p_{s o}$. When $p \geq p^{*}$, Miyamoto used the technique of phase plane analysis to show that $u_{\mu}(r) / u_{s}(r) \rightarrow 1$ as $r \rightarrow \infty$ (cf. Lemma 2.5 in [20]). When $p \geq p_{s o}$, Theorem 1.2 shows that the decay rate of $u_{\mu}$ is the same as that of $u_{s}$. Furthermore, if $\lim _{r \rightarrow \infty} u(r) r^{-\frac{2 k}{p-k}}$ exists, then it must be $A$ which is introduced in (1.5). In fact, integrating (1.4) twice yields

$$
u(r)=u(0)-\left(\frac{k}{C_{n-1}^{k-1}}\right)^{1 / k} \int_{0}^{r}\left[t^{k-n} \int_{0}^{t} s^{n-1} u^{p}(s) d s\right]^{1 / k} d t .
$$

Write $B:=\lim _{r \rightarrow \infty} \frac{u(r)}{r \frac{2 k}{p-k}}$. Using the L'Hospital principle twice, we get

$$
B^{k}=\frac{k}{C_{n-1}^{k-1}}\left(\frac{2 k}{p-k}\right)^{-k} \frac{\int_{0}^{r} s^{n-1} u^{p}(s) d s}{r^{n-\frac{2 p k}{p-k}}}=\frac{k}{C_{n-1}^{k-1}}\left(\frac{2 k}{p-k}\right)^{-k}\left(n-\frac{2 p k}{p-k}\right)^{-1} B^{p},
$$

which implies $B=A$.

\section{Stable solutions}

\subsection{Proof of Theorem 1.3}

Step 1. We claim that for every $\gamma \in\left[1, \frac{2 p+2 \sqrt{p(p-k)}-k}{k}\right)$ and any integer $m \geq$ $\max \left\{\frac{p+\gamma}{p-k}, 2\right\}$, there exists a constant $C>0$ such that for any $\psi \in W_{*}$, there holds

$$
\int_{0}^{R} r^{n-1} u^{p+\gamma} \psi^{m(k+1)} d r \leq C \int_{0}^{R}\left(r^{\frac{(n-k)(p+\gamma)-(n-1)(\gamma+k)}{p+\gamma}}\left|\psi^{\prime}\right|^{k+1}\right)^{\frac{p+\gamma}{p-k}} d r .
$$

Proof of (3.1). Let $\psi \in W_{*}$ be a cut-off function such that $0 \leq \psi \leq 1$ and

$$
\psi(r)= \begin{cases}1, & \text { if } r \leq R / 2, \\ 0, & \text { if } r \geq R\end{cases}
$$

Clearly, there exists a constant $C>0$ such that $\left|\psi^{\prime}\right| \leq \frac{C}{R}$.

Taking $\varphi=u^{\gamma} \psi^{m(k+1)}$ in (1.11), we get

$$
\begin{aligned}
& \frac{\gamma}{k} C_{n-1}^{k-1} \int_{0}^{R} r^{n-k}\left|u^{\prime}\right|^{k+1} u^{\gamma-1} \psi^{m(k+1)} d r \\
& \quad \leq \frac{k+1}{k} C_{n-1}^{k-1} \int_{0}^{R} r^{n-k}\left|u^{\prime}\right|^{k} u^{\gamma} \psi^{m k}\left|\left(\psi^{m}\right)^{\prime}\right| d r+\int_{0}^{R} r^{n-1} u^{p+\gamma} \psi^{m(k+1)} d r .
\end{aligned}
$$

Using the Young inequality to the first term of the right hand side, we can obtain that for any small $\varepsilon>0$,

$$
\begin{aligned}
& \left(\frac{\gamma}{k} C_{n-1}^{k-1}-\varepsilon^{2}\right) \int_{0}^{R} r^{n-k} u^{\gamma-1}\left|u^{\prime}\right|^{k+1} \psi^{m(k+1)} d r \\
& \quad \leq C_{\varepsilon} \int_{0}^{R} r^{n-k} u^{\gamma+k}\left|\left(\psi^{m}\right)^{\prime}\right|^{k+1} d r+\int_{0}^{R} r^{n-1} u^{p+\gamma} \psi^{m(k+1)} d r .
\end{aligned}
$$


Taking $\varphi=u^{\frac{\gamma+1}{2}} \psi^{\frac{m(k+1)}{2}}$ in (1.12), we have

$$
\begin{aligned}
& p \int_{0}^{R} r^{n-1} u^{p+\gamma} \psi^{m(k+1)} d r \\
& \leq \frac{C_{n-1}^{k-1}(\gamma+1)^{2}}{4} \int_{0}^{R} r^{n-k} u^{\gamma-1}\left|u^{\prime}\right|^{k+1} \psi^{m(k+1)} d r \\
& +\frac{C_{n-1}^{k-1}(k+1)^{2}}{4} \int_{0}^{R} r^{n-k}\left|u^{\prime}\right|^{k-1} u^{\gamma+1} \psi^{m(k-1)}\left|\left(\psi^{m}\right)^{\prime}\right|^{2} d r \\
& +\frac{C_{n-1}^{k-1}(\gamma+1)(k+1)}{2} \int_{0}^{R} r^{n-k}\left|u^{\prime}\right|^{k} u^{\gamma} \psi^{m k}\left|\left(\psi^{m}\right)^{\prime}\right| d r .
\end{aligned}
$$

Using the Young inequality to the second and the third terms of the right hand side of (3.3), we get

$$
\begin{aligned}
& p \int_{0}^{R} r^{n-1} u^{p+\gamma} \psi^{m(k+1)} d r \\
\leq & \left(\frac{C_{n-1}^{k-1}(\gamma+1)^{2}}{4}+\varepsilon^{2}\right) \int_{0}^{R} r^{n-k}\left|u^{\prime}\right|^{k+1} u^{\gamma-1} \psi^{m(k+1)} d r \\
+ & C_{\varepsilon} \int_{0}^{R} r^{n-k}\left|\left(\psi^{m}\right)^{\prime}\right|^{k+1} u^{\gamma+k} d r .
\end{aligned}
$$

Combining (3.2) and (3.4), we obtain by the Hölder inequality that

$$
\begin{aligned}
& {\left[p-\left(\frac{C_{n-1}^{k-1}(\gamma+1)^{2}}{4}+\varepsilon^{2}\right) \frac{1}{\frac{\gamma}{k} C_{n-1}^{k-1}-\varepsilon^{2}}\right] \int_{0}^{R} r^{n-1} u^{p+\gamma} \psi^{m(k+1)} d r} \\
& \leq C \int_{0}^{R} r^{n-k}\left|\left(\psi^{m}\right)^{\prime}\right|^{k+1} u^{\gamma+k} d r \\
& \leq C\left[\int_{0}^{R}\left(r^{(n-1) \frac{\gamma+k}{p+\gamma}} u^{\gamma+k} \psi^{(m-1)(k+1)}\right)^{\frac{p+\gamma}{\gamma+k}} d r\right]^{\frac{\gamma+k}{p+\gamma}} \\
& \quad \cdot\left[\int_{0}^{R}\left(r^{\frac{(n-k)(p+\gamma)-(n-1)(\gamma+k)}{p+\gamma}}\left|\psi^{\prime}\right|^{k+1}\right)^{\frac{p+\gamma}{p-k}} d r\right]^{\frac{p-k}{p+\gamma}} .
\end{aligned}
$$

In view of $\gamma \in\left[1, \frac{2 p+2 \sqrt{p(p-k)}-k}{k}\right), \lim _{\varepsilon \rightarrow 0}\left[p-\left(\frac{C_{n-1}^{k-1}(\gamma+1)^{2}}{4}+\varepsilon^{2}\right) \frac{1}{\frac{\gamma}{k} C_{n-1}^{k-1}-\varepsilon^{2}}\right]=p-$ $\frac{k(\gamma+1)^{2}}{4 \gamma}>0$. Therefore, the coefficient of the left hand side of (3.5) is positive as long as $\varepsilon$ is sufficiently small. Therefore, noting $(m-1)(k+1) \frac{p+\gamma}{\gamma+k} \geq m(k+1)$ which is implied by $m \geq \max \left\{\frac{p+\gamma}{p-k}, 2\right\}$, we can deduce (3.1) from (3.5) by the Young inequality.

Step 2. By the definition of $\psi$, from (3.1) we can deduce that

$$
\int_{0}^{R} r^{n-1} u^{p+\gamma} \psi^{m(k+1)} d r \leq C R^{n+1-\frac{(2 k+1)(p+\gamma)-(\gamma+k)}{p-k}} .
$$

When $n+1-\frac{(2 k+1)(p+\gamma)-(\gamma+k)}{p-k}<0$, the desired claim follows by letting $R \rightarrow \infty$.

Consider a real-valued function

$$
f(t)=\frac{(2 k+1)(t+\gamma(t))-(\gamma(t)+k)}{t-k}, \quad t \in(k, \infty),
$$


where $\gamma(t)=\frac{2 t+2 \sqrt{t(t-k)}-k}{k}$. Clearly, we know $f(t)$ is a strictly decreasing function (by virtue of $f^{\prime}(t)<0$ on $(k, \infty)$ ), satisfying $\lim _{t \rightarrow k} f(t)=\infty$ and $\lim _{t \rightarrow \infty} f(t)=2 k+9$. Therefore, we consider separately two cases: $n \leq 2 k+8$, and $n \geq 2 k+9$.

Case I: $n \leq 2 k+8$. In view of $p>p_{s e}$, there exists $\gamma \in\left[1, \frac{2 p+2 \sqrt{p(p-k)}-k}{k}\right)$ such that $n+1-\frac{(2 k+1)(p+\gamma)-(\gamma+k)}{p-k}<0$ is true.

Case II: $n \geq 2 k+9$. In view of $p>p_{s e}$, there exists a unique $p_{0}>k$ such that $n+1=f\left(p_{0}\right)$ since $f(t)$ is decreasing in $(k, \infty)$. Therefore, $p_{0}$ satisfies

$$
(n-2 k)(n-2 k-8) p_{0}^{2}-2 k\left[n^{2}-2(k+3) n+4 k\right] p_{0}+k^{2}(n-2)^{2}=0,
$$

and

$$
(n-2 k-4) p_{0}-(n-2) k>4\left(p_{0}-k\right) .
$$

The roots of equation (3.7) are

$$
\begin{aligned}
& p_{1}=\frac{k\left[n^{2}-2(k+3) n+4 k\right]+4 k \sqrt{2(k+1) n-4 k}}{(n-2 k)(n-2 k-8)}, \\
& p_{2}=\frac{k\left[n^{2}-2(k+3) n+4 k\right]-4 k \sqrt{2(k+1) n-4 k}}{(n-2 k)(n-2 k-8)} .
\end{aligned}
$$

Inequality (3.8) implies $p_{0}>p_{2}$, and hence we take $p_{0}=p_{1}$ (it equals exactly $\left.p_{j l}\right)$. Thus, when $p<p_{j l}$, there exists $\gamma \in\left[1, \frac{2 p+2 \sqrt{p(p-k)}-k}{k}\right)$ satisfying $n+1-$ $\frac{(2 k+1)(p+\gamma)-(\gamma+k)}{p-k}<0$.

No matter in Case I or Case II, letting $R \rightarrow \infty$ in (3.6), we can deduce $\int_{0}^{R} r^{n-1} u^{p+\gamma} d r \rightarrow 0$. This contradiction shows that (1.4) has no positive stable solution as long as $p<p_{j l}$.

\subsection{Proof of Theorem 1.4}

Let $u_{s}$ be the singular solution of (1.4) given by (1.5). We will prove that the singular solution $u_{s}(r)$ is stable when $n \geq 2 k+9$ and $p \geq p_{j l}$.

First, we claim that $u_{s}$ satisfies (1.11). In fact, by (1.7), the improper integral $\int_{0}^{\infty} r^{n-1} u_{s}^{p} \varphi d r \leq C \int_{0}^{R} r^{n-1-\frac{2 p k}{p-k}} d r<\infty$. Similarly, the left hand side of (1.11) also makes sense. In addition, $u_{s}$ solves (1.4). Multiply by the test function $\varphi \in W_{*}$ and integrate from 0 to $\infty$. Noting $r^{n-k}\left|u_{s}^{\prime}(r)\right|^{k} \rightarrow 0$ as $r \rightarrow 0$, we know that the claim is true.

To prove that $u_{s}$ satisfies (1.12), we observe firstly that

$$
\begin{aligned}
& p\left(\frac{2}{p-k}\right)\left(n-\frac{2 p k}{p-k}\right) \leq \frac{\left(n-2-\frac{2 p(k-1)}{p-k}\right)^{2}}{4} \\
& \Leftrightarrow 8 n\left(p^{2}-k p\right)-16 k p^{2} \leq(n-2)^{2}\left(p^{2}-2 k p+k^{2}\right) \\
& \quad+4(k-1)^{2} p^{2}-4(k-1)(n-2)\left(p^{2}-k p\right) \\
& \Leftrightarrow(n-2 k)(n-2 k-8) p^{2}-2 k\left(n^{2}-2(k+3) n+4 k\right) p \\
& \quad+k^{2}(n-2)^{2} \geq 0 \\
& \Leftrightarrow p \in\left(-\infty, p_{2}\right] \bigcup\left[p_{j l},+\infty\right)
\end{aligned}
$$


where $p_{2}$ is defined in (3.10). On the other hand, by Definition 1.2 , we have that for any $\phi \in C_{c}^{\infty}\left(R^{n}\right)$,

$$
\begin{aligned}
& C_{n-1}^{k-1} \int_{R^{n}} \frac{1}{|x|^{k-1}}\left|u_{s}^{\prime}\right|^{k-1}|\nabla \phi|^{2} d x-p \int_{R^{n}} u_{s}^{p-1} \phi^{2} d x \\
& =C_{n-1}^{k-1} \int_{R^{n}}\left(\frac{1}{k} C_{n-1}^{k-1}\right)^{\frac{k-1}{p-k}}\left(\frac{2 k}{p-k}\right)^{\frac{(k-1) p}{p-k}}\left(n-\frac{2 p k}{p-k}\right)^{\frac{k-1}{p-k}} \frac{1}{|x|^{\frac{2 p(k-1)}{p-k}}}|\nabla \varphi|^{2} d x \\
& \quad-p \int_{R^{n}}\left(\frac{1}{k} C_{n-1}^{k-1}\right)^{\frac{p-1}{p-k}}\left(\frac{2 k}{p-k}\right)^{\frac{(p-1) k}{p-k}}\left(n-\frac{2 p k}{p-k}\right)^{\frac{p-1}{p-k}} \frac{1}{|x|^{\frac{2(p-1) k}{p-k}}} \varphi^{2} d x \\
& =C_{0}\left(\int_{R^{n}} \frac{1}{|x|^{\frac{2 p(k-1)}{p-k}}}|\nabla \phi|^{2} d x-p\left(\frac{2}{p-k}\right)\left(n-\frac{2 p k}{p-k}\right) \int_{R^{n}} \frac{1}{|x|^{\frac{2(p-1) k}{p-k}}} \phi^{2}\right) d x,
\end{aligned}
$$

where

$$
C_{0}=C_{n-1}^{k-1}\left(\frac{1}{k} C_{n-1}^{k-1}\right)^{\frac{k-1}{p-k}}\left(\frac{2 k}{p-k}\right)^{\frac{(k-1) p}{p-k}}\left(n-\frac{2 p k}{p-k}\right)^{\frac{k-1}{p-k}} .
$$

By $p \geq p_{j l}$, (3.11) implies that

$$
\begin{aligned}
& \int_{R^{n}} \frac{1}{|x|^{\frac{2 p(k-1)}{p-k}}}|\nabla \phi|^{2} d x-p\left(\frac{2}{p-k}\right)\left(n-\frac{2 p k}{p-k}\right) \int_{R^{n}} \frac{1}{|x|^{\frac{2(p-1) k}{p-k}}} \phi^{2} d x \\
& \geq \int_{R^{n}} \frac{1}{|x|^{\frac{2 p(k-1)}{p-k}}}|\nabla \phi|^{2} d x-\frac{\left(n-2-\frac{2 p(k-1)}{p-k}\right)^{2}}{4} \int_{R^{n}} \frac{1}{|x|^{\frac{2(p-1) k}{p-k}}} \phi^{2} d x .
\end{aligned}
$$

It follows that

$$
Q_{u_{s}}(\varphi)>0, \quad \forall \varphi \in W_{*}
$$

by the Caffarelli-Kohn-Nirenberg inequality (cf. 4])

$$
\int_{R^{n}} \frac{|\nabla \phi|^{2}}{|x|^{2 a}} d x>C_{a, b} \int_{R^{n}} \frac{\phi^{2}}{|x|^{2 b}} d x, \quad \forall \phi \in D_{a}^{1,2}\left(R^{n}\right),
$$

where $n \geq 3,0 \leq a<\frac{n-2}{2}$ and $a \leq b \leq a+1$, the best constant $C_{a, b}$ is given by $C_{a, b}=\frac{(n-2-2 a)^{2}}{4}$. Here we take $a=\frac{p(k-1)}{p-k}$ and $b=a+1$. This result shows that $u_{s}$ is a stable solution of (1.4) when $n \geq 2 k+9$ and $p \geq p_{j l}$. The proof of Theorem 1.4 is complete.

\subsection{Proof of Theorem 1.5}

Step 1. When $p=p_{s o}$, all regular solutions $u_{\rho}$ of (1.6) can be written as the form given by (1.8). When $r$ is suitably large,

$$
u_{\rho}(r) \leq D_{1} r^{-\frac{n-2 k}{k}}, \quad\left|u_{\rho}^{\prime}\right| \geq D_{2} r^{-\frac{n-k}{k}},
$$

where $D_{1}, D_{2}$ are positive constants independent of $r$. Thus,

$$
p u^{p-1}(r)=O\left(r^{-\frac{(k-1) n}{k}-4}\right), \quad \text { as } r \rightarrow \infty .
$$

Therefore, we can find some $R>0$ such that for all $|x|>R$ and $\phi \in C_{c}^{\infty}\left(R^{n} \backslash\right.$ $\left.\overline{B_{R}(0)}\right)$, there holds

$$
p u_{\rho}^{p-1}(|x|) \phi^{2}(x)<C^{*}|x|^{-\frac{(k-1) n}{k}-2} \phi^{2}(x),
$$


where $C^{*}=\frac{\left(n-2-\frac{k-1}{k} n\right)^{2}}{4} D_{2}^{k-1} C_{n-1}^{k-1}\left(\frac{n-2 k}{k}\right)^{k-1}$. Thus,

$$
\begin{aligned}
C_{n-1}^{k-1} \int_{R^{n}} \frac{1}{|x|^{k-1}}\left|u_{\rho}^{\prime}\right|^{k-1}|\nabla \phi|^{2} d x-p \int_{R^{n}} u_{\rho}^{p-1} \phi^{2} d x \\
\geq D_{2}^{k-1} C_{n-1}^{k-1}\left(\frac{n-2 k}{k}\right)^{k-1} \int_{R^{n}} \frac{1}{|x|^{\frac{k-1}{k}} n}|\nabla \phi|^{2} d x \\
\quad-C^{*} \int_{R^{n}} \frac{1}{|x|^{\frac{k-1}{k} n+2}} \phi^{2} d x \\
=D_{2}^{k-1} C_{n-1}^{k-1}\left(\frac{n-2 k}{k}\right)^{k-1}\left(\int_{R^{n}} \frac{1}{|x|^{\frac{k-1}{k}} n}|\nabla \phi|^{2} d x\right. \\
\left.\quad-\frac{\left(n-2-\frac{k-1}{k} n\right)^{2}}{4} \int_{R^{n}} \frac{1}{|x|^{\frac{k-1}{k} n+2}} \phi^{2}\right) d x,
\end{aligned}
$$

and the right hand side is nonnegative by the Caffarelli-Kohn-Nirenberg inequality (3.14) with $a=\frac{k-1}{2 k} n$ and $b=a+1$. Therefore, $Q_{u_{\rho}}(\varphi) \geq 0$ for every $\varphi \in C_{c}^{\infty}(R, \infty)$. In addition, $u_{\rho}$ also satisfies (1.11). So the regular solution $u_{\rho}$ is stable on $(R, \infty)$.

Step 2. Let $u_{\mu}$ (see (1.9)) be a regular solution of (1.6) with $p \geq \max \left\{p^{*}, p_{j l}\right\}$. We claim that $u_{\mu}$ is stable on $(R, \infty)$ for some $R>0$.

We at first prove $\lim _{r \rightarrow \infty} u_{\mu}^{\prime}(r) / u_{s}^{\prime}(r)=1$ when $p \geq \max \left\{p^{*}, p_{j l}\right\}$.

Clearly, $u_{s}^{\prime}=-\left(\frac{1}{k} C_{n-1}^{k-1}\right)^{\frac{1}{p-k}}\left(\frac{2 k}{p-k}\right)^{\frac{p}{p-k}}\left(n-\frac{2 p k}{p-k}\right)^{\frac{1}{p-k}} r^{-\frac{p+k}{p-k}}$.

Combining with (2.12) and using the L'Hospital principle, we get

$$
\begin{aligned}
\lim _{r \rightarrow \infty}\left(\frac{u_{\mu}^{\prime}}{u_{s}^{\prime}}\right)^{k} & =\lim _{r \rightarrow \infty} \frac{\int_{0}^{r} s^{n-1} u_{\mu}^{p}(s) d s}{\left(\frac{1}{k} C_{n-1}^{k-1}\right)^{\frac{p}{p-k}}\left(\frac{2 k}{p-k}\right)^{\frac{p k}{p-k}}\left(n-\frac{2 p k}{p-}\right)^{\frac{k}{p-k}} r^{n-\frac{2 p k}{p-k}}} \\
& =\lim _{r \rightarrow \infty} \frac{r^{n-1} u_{\mu}^{p}(r)}{\left(\frac{1}{k} C_{n-1}^{k-1}\right)^{\frac{p}{p-k}}\left(\frac{2 k}{p-k}\right)^{\frac{p k}{p-k}}\left(n-\frac{2 p k}{p-k}\right)^{\frac{p}{p-k}} r^{n-\frac{2 p k}{p-k}-1}} \\
& =\lim _{r \rightarrow \infty} \frac{u_{\mu}^{p}(r)}{u_{s}^{p}(r)} .
\end{aligned}
$$

By Remark 2.1, there holds $\lim _{r \rightarrow \infty} u_{\mu}^{\prime}(r) / u_{s}^{\prime}(r)=1$ when $p \geq \max \left\{p^{*}, p_{j l}\right\}$. Thus, there exists sufficiently large $R>0$ such that as $r>R$,

$$
\left|u_{\mu}^{\prime}(r)\right|^{k-1}=\left|u_{s}^{\prime}(r)\right|^{k-1}+o(1) r^{-\frac{(k-1)(p+k)}{p-k}} .
$$

Therefore, by the strict inequality (3.13), we can find a suitably small $\delta_{0}>0$ such that for any $\psi \in C_{c}^{\infty}\left(R^{n} \backslash \overline{B_{R}(0)}\right)$,

$$
\begin{aligned}
& C_{n-1}^{k-1} \int_{R^{n}} \frac{\left|u_{\mu}^{\prime}(|x|)\right|^{k-1}}{|x|^{k-1}}|\nabla \phi|^{2} d x \\
& =C_{n-1}^{k-1} \int_{R^{n}} \frac{\left|u_{s}^{\prime}(|x|)\right|^{k-1}+o(1)|x|^{-\frac{(k-1)(p+k)}{p-k}}}{|x|^{k-1}}|\nabla \phi|^{2} d x \\
& \geq C_{0}\left[p\left(\frac{2}{p-k}\right)\left(n-\frac{2 p k}{p-k}\right)+\delta_{0}+o(1)\right] \int_{R^{n}} \frac{1}{|x|^{\frac{2(p-1) k}{p-k}}} \phi^{2} d x \\
& \geq p \int_{R^{n}} u_{s}(|x|)^{p-1} \phi^{2} d x .
\end{aligned}
$$


Here $C_{0}$ is the constant in (3.12). In view of $u_{s}(r)>u_{\mu}(r)$ for $r>R$ (see Remark 1.3), we can see $Q_{u_{\mu}}(\varphi) \geq 0$ for any $\varphi \in C_{c}^{\infty}(R, \infty)$. In addition, $u_{\mu}$ satisfies (1.11). Thus, $u_{\mu}$ is stable on $(R, \infty)$ for some $R>0$.

Step 3. Let $U_{s}$ be a singular solution of (1.4) with $p \in\left(p_{s e}, p_{s o}\right)$ introduced in Remark 1.1. By the same as in the proof of Theorem 1.4 $U_{s}$ still satisfies (1.11) since 0 is not the singular point in the improper integrals of (1.11) which is implied by $\lim _{r \rightarrow 0} U_{s}(r) / u_{s}(r)=1$.

In addition, by an analogous argument in Step 1, $U_{s}$ still satisfies (1.12). In fact, $\lim _{r \rightarrow \infty} U_{s}(r) r^{\frac{n-2 k}{k}}=\lambda$ implies

$$
U_{s}(r) \leq C r^{-\frac{n-2 k}{k}} \quad \text { for large } r .
$$

On the other hand, by (2.12), the monotonicity of $U_{s}$, and (3.17), there holds

$$
\left|U_{s}^{\prime}\right|^{k} \geq c r^{k-n} U_{s}^{p}(r) \int_{0}^{r} s^{n-1} d s \geq c r^{k-p \frac{n-2 k}{k}}
$$

for large $r$. Therefore, applying the Caffarelli-Kohn-Nirenberg inequality (3.14) with $a=p \frac{n-2 k}{k} \frac{k-1}{2 k}$ and $b=a+1$, we obtain by (3.17) and $p>p_{\text {se }}$ that

$$
\begin{aligned}
& \int_{R^{n}} \frac{\left|U_{s}^{\prime}(|x|)\right|^{k-1}}{|x|^{k-1}}|\nabla \phi|^{2} d x \geq c \int_{R^{n}} \frac{\phi^{2} d x}{|x|^{p \frac{n-2 k}{k} \frac{k-1}{k}+2}} \\
& \geq c \int_{R^{n}} \frac{\phi^{2} d x}{|x|^{(p-1) \frac{n-2 k}{k}}} \geq p \int_{R^{n}} U_{s}^{p-1}(|x|) \phi^{2} d x
\end{aligned}
$$

for any $\phi \in C_{c}^{\infty}\left(R^{n} \backslash \overline{B_{R}(0)}\right)$ with suitably large $R$.

\section{References}

[1] L. Caffarelli, L. Nirenberg, J. Spruck, The Dirichlet problem for nonlinear second elliptic equations, III. Functions of the eigenvalues of the Hessian, Acta Math., 155 (1985), 261-301.

[2] W. Chen, C. Li, Classification of solutions of some nonlinear elliptic equations, Duke Math. J., 63 (1991), 615-622.

[3] W. Chen, C. Li, Radial symmetry of solutions for some integral systems of Wolff type, Discrete Contin. Dyn. Syst., 30 (2011), 1083-1093.

[4] K. Chou, C. Chu, On the best constant for a weighted Sobolev-Hardy inequality, J. London Math. Soc., 2 (1993), 137-151.

[5] P. Clément, D. de Figueiredo, and E. Mitidieri, Quasilinear elliptic equations with critical exponents, Topol. Methods Nonlinear Anal., 7 (1996), 133-170.

[6] L. Damascelli, A. Farina, B. Sciunzi, E. Valdinoci, Liouville results for $m$-Laplace equations of Lane-Emden-Fowler type, Ann. Inst. H. Poincare Anal. Non Lineaire, 26 (2009), 1099-1119.

[7] E. N. Dancer, Stable solutions on $R^{n}$ and the primary branch of some non-self-adjoint convex problems, Differential Integral Equations 17 (2004), 961-970. 
[8] A. Farina, On the classification of solutions of the Lane-Emden equation on unbounded domains of $R^{n}$, J. Math. Pures Appl., 87 (2007), 537-561.

[9] B. Gidas, J. Spruck, Global and local behavior of positive solutions of nonlinear elliptic equations, Comm. Pure Appl. Math., 34 (1981), 525-598.

[10] C. Gui, W.-M. Ni, X.-F. Wang, On the stability and instability of positive steady states of a semilinear heat equation in $R^{n}$, Comm. Pure Appl. Math., 45 (1992), 1153-1181.

[11] Z. Guo, J. Wei, Global solution branch and Morse index estimates of a semilinear elliptic equation with super-critical exponent, Trans. Amer. Math. Soc., 363 (2011), 4777-4799.

[12] N.M. Ivochkina, O.A. Ladyzhenskaya, The first initial-boundary value problem for evolution equations generated by traces of order $m$ of the hessian of the unknown surface, Russian Acad. Sci. Dokl. Math., 50 (1995), 61-65.

[13] D. Joseph, T. Lundgren, Quasilinear Dirichlet problems driven by positive sources, Arch. Rational Meth. Anal., 49 (1972/73), 241-269.

[14] D. Labutin, Potential estimates for a class of fully nonlinear elliptic equations, Duke Math. J., 111 (2002), 1-49.

[15] Y. Lei, Critical conditions and finite energy solutions of several nonlinear elliptic PDEs in $R^{n}$, J. Differential Equations, 258 (2015), 4033-4061.

[16] Y. Lei, C. Li, Integrability and asymptotics of positive solutions of a $\gamma$ Laplace system, J. Differential Equations, 252 (2012), 2739-2758.

[17] Y. Lei, C. Li, Sharp criteria of Liouville type for some nonlinear systems, Discrete Contin. Dyn. Syst., 36 (2016), 3277-3315.

[18] Y. Li, W.-M. Ni, On conformal scalar curvature equations in $R^{n}$, Duke Math. J., 57 (1988), 895-924.

[19] C. Ma, W. Chen, C. Li, Regularity of solutions for an integral system of Wolff type, Adv. Math., 226 (2011), 2676-2699.

[20] Y. Miyamoto, Intersection properties of radial solutions and global bifurcation diagrams for supercritical quasilinear elliptic equations, Nonlinear Diff. Equ. Appl.(NoDEA), to appear.

[21] W.-M. Ni, On the elliptic equation $\Delta u+K(x) u^{(n+2) /(n-2)}=0$, its generalizations, and applications in geometry, Indiana Univ. Math. J., 31 (1982), 493-529.

[22] Q. Ou, Nonexistence results for Hessian inequality, Methods Appl. Anal., 17 (2010), 213-224.

[23] N. Phuc, I. Verbitsky, Quasilinear and Hessian equations of Lane-Emden type, Ann. Math., 168 (2008), 859-914.

[24] C. Ren, The first initial-boundary value problem for fully nonlinear parabolic equations generated by functions of the eigenvalues of the Hessian, J. Math. Anal. Appl., 339 (2009), 1362-1373. 
[25] J. Serrin, H. Zou, Cauchy-Liouville and universal boundedness theorems for quasilinear elliptic equations and inequalities, Acta Math., 189 (2002), 79-142.

[26] S. Sun, Y. Lei, Fast decay estimates for integrable solutions of the LaneEmden type integral systems involving the Wolff potentials, J. Funct. Anal., 263 (2012), 3857-3882.

[27] G.-J. Tian, X.-J. Wang, Moser-Trudinger type inequalities for the Hessian equation, J. Funct. Anal., 259 (2010), 1974-2002.

[28] K. Tso, Remarks on critical exponents for Hessian operators, Ann. Inst. H. Poincare Anal. Non Lineaire, 7 (1990), 113-122.

[29] J. Villavert, A characterization of fast decaying solutions for quasilinear and Wolff type systems with singular coefficients, J. Math. Anal. Appl., 424 (2015), 1348-1373.

[30] G. Wang, H. Liu, Some results on evolutionary equations involving functions of the eigenvalues of the Hessian, Northeast. Math. J., 13 (1997), 433-448.

[31] X.-F. Wang, On the Cauchy problem for reaction-diffusion equations, Trans. Amer. Math. Soc., 337 (1993), 549-590.

[32] X.-J. Wang, A class of fully nonlinear elliptic equations and related functionals, Indiana Univ. Math. J., 43 (1994), 25-54. 